\title{
On several species of the millipede genus Eustrongylosoma Silvestri, 1896 from Vanuatu, Melanesia, southwestern Pacific (Diplopoda: Polydesmida: Paradoxosomatidae)
}

\section{О нескольких видах диплопод рода Eustrongylosoma Silvestri, 1896 из Вануату (Меланезия, юго-запад Тихого океана) (Diplopoda: Polydesmida: Paradoxosomatidae)}

\author{
S.I. Golovatch ${ }^{1}$, J.-J. Geoffroy ${ }^{2}$ \\ С.И. Головач ${ }^{1}$, Ж.-Ж. Жоффруа ${ }^{2}$
}

\begin{abstract}
${ }^{1}$ Institute for Problems of Ecology and Evolution, Russian Academy of Sciences, Leninsky prospekt 33, Moscow 119071 Russia.
${ }^{1}$ Институт проблем экологии и эволюции РАН, Ленинский проспект, 33, Москва 119071 Россия.

${ }^{2}$ Muséum national d'Histoire naturelle, Département Ecologie \& Gestion de la Biodiversité, UMR 7204 CESCO, 4, avenue du Petit Château, F-91800 Brunoy, France.
\end{abstract}

KEY WORDS: Diplopoda, Paradoxosomatidae, Eustrongylosoma, new species, Vanuatu.

КЛЮЧЕВЫЕ СЛОВА: Diplopoda, Paradoxosomatidae, Eustrongylosoma, новый вид, Вануату.

ABSTRACT. Four new species of the large, mostly Papuan genus Eustrongylosoma are described from the Espiritu Santo Island, Vanuatu: E. hirsutum sp.n., E. vanuatu sp.n., E. acutum sp.n. and E. picturatum sp.n. These are only some of the species of the genus that actually populate the island. Besides Eustrongyloso$m a$, Espiritu Santo seems to fail to support any other presumed endemics from the family Paradoxosomatidae. Despite some encounters in caves, none of the species demonstrates obvious troglomorphic traits.

РЕЗЮМЕ. С острова Эспириту-Санто (Вануату) описаны четыре новых вида большого, в основном папуасского рода Eustrongylosoma: E. hirsutum sp.n., E. vanuatu sp.n., E. acutum sp.n. и E. picturatum sp.n. Это лишь несколько из видов этого рода, на самом деле живущих на этом острове. Кроме Eustrongylosoma, иных предположительно эндемичных видов из семейства Paradoxosomatidae на ЭспиритуСанто, кажется, нет. Нусмотря на несколько находок в пещерах, ни один из видов не обнаруживает очевидные трогломорфные признаки.

\section{Introduction}

The family Paradoxosomatidae is long known to dominate the Indo-Australian millipede fauna, including that of New Guinea together with most of the adjacent archipelagos [Jeekel, 1968]. The large genus Eustrongylosoma Silvestri, 1896 currently comprises 29 species described from New Guinea (22), the Solomon Islands (5), Sulawesi and Borneo (1), and the Philippines (1, Luzon Island) [Golovatch \& Stoev, 2013]. A few more, still undescribed Eustrongylosoma species have been reported from the Caroline island Ponape, Micronesia, as well as from Mindanao, Philippines, and Biak Island, off New Guinea [Hoffman, 1977/78; Golovatch, 1997].

The present note puts on record a few more new congeners from Espiritu Santo, Vanuatu, Melanesia. Surprisingly, this small and remote archipelago shows a comparable if not greater diversity of Eustrongyloso$m a$ species than the far larger Solomon Islands [Jeekel, 1973] which, in addition, lie much closer to New Guinea, a presumed major source area. This discrepancy seems easily to be accounted for by our still highly deficient knowledge of the very rich and diverse millipede faunas of New Guinea (with its numerous adjacent archipelagos) and Melanesia. On the other hand, the Loyalty Islands, lying between the Gondwana-derived New Caledonia and Vanuatu, yet much closer to New Caledonia, support a diplopod fauna which is most strongly influenced by that of New Caledonia, including a complete lack of indigenous Paradoxosomatidae [Brolemann, 1931]. Similarly, unlike Vanuatu, the Fiji Islands appear to be totally devoid of endemic paradoxosomatids as well [Jeekel, 1980].

Only six millipede species have hitherto been reported from Vanuatu, four of which are definitely introduced, pantropical "tramp" elements. Yet two seem to be endemic: Lobiferodesmus vanuatu Golovatch, Geoffroy, Mauriès et VandenSpiegel, 2008 (Polydesmida: Pyrgodesmidae) and Eutrichodesmus communicans Golovatch, Geoffroy, Mauriès et VandenSpiegel, 2009 (Polydesmida: Haplodesmidae) [Golovatch et al., 2008, 2009]. One more species of Pyrgodesmidae, presumably also endemic to Espiritu Santo, is shortly to be described elsewhere. Most of the species, including the 
new ones described herein, have been collected during the famous SANTO 2006 expedition held by the Muséum national d'Histoire naturelle, Paris, France [Bouchet et al., 2009, 2011, 2012]. The present note addresses only a few of the Eustrongylosoma samples taken in Vanuatu. For this reason alone, no local key is attempted here as obviously too premature.

\section{Material and methods}

Material was collected in 2005 and 2006, mostly during the SANTO 2006 Global Biodiversity Survey which focused on sampling the biota of the Espiritu Santo Island, Vanuatu. Millipedes were mainly taken by Group "Karst" which included Louis Deharveng, Anne Bedos (MNHN), Cahyo Rahmadi (Museum Zoologicum Bogoriense, Cibinong, Java, Indonesia), Josiane Lips (Villeurbanne, France) and several other persons, and as well as Christine Rollard and Claire Villemant (MNHN), who represented Group "Forest". Hand collection was combined with Berlese extractions, the latter techniques having allowed for the bulk of smaller millipedes to be taken, including some Eustrongylosoma.

All holotypes and most of the paratypes are housed in the Muséum national d'Histoire naturelle (MNHN), Paris, France, with only a few paratypes shared with the collections of the Zoological Museum, State University of Moscow (ZMUM), Moscow, Russia, and the Natural History Museum of Denmark (ZMUC), Copenhagen, Denmark.

\section{Taxonomic part}

\section{Eustrongylosoma hirsutum sp.n.} Figs 1-11.

HOLOTYPE O (MNHN JA 133), Vanuatu, Espiritu Santo Island, Penaoru, 900 m a.s.l., litter, Berlese extraction, 12.11.2006, leg. C. Villemant (VAN06-06).

PARATYPE: $1 O^{7}$ (MNHN JA 133), same data, together with holotype

NAME. To emphasize the densely and irregularly hirsute metaterga.

DIAGNOSIS. This new species joins the few congeners like E. setosum (Chamberlin, 1920), E. gracile (Chamberlin, 1920), E. reductum (Chamberlin, 1920), all three from the Solomon Islands [see Jeekel, 1973], as well as E. papua Golovatch et Stoev, 2011 and E. beroni Golovatch et Stoev, 2009, both latter species from Papua New Guinea [Golovatch \& Stoev, 2009, 2011], in showing particularly abundant and irregular pilosity on the metaterga. E. hirsutum sp.n. differs from all of them, however, in the posterior corner of all postcollum paraterga being acute and projecting behind the tergal margin, combined with the paraterga being devoid of lateral indentations (Figs 3-5), the apicolateral lobe (I) on the gonofemorite small and dentiform, and the solenophore deeply bifid (Figs 9-11).
DESCRIPTION. Length ca $11 \mathrm{~mm}$, width of midbody pro- and metazona 0.9 and $1.2 \mathrm{~mm}$, respectively $\left(O^{7}\right)$. Coloration light grey-yellow to yellow-red-brown (Figs 1-3). Pattern clear due to a broad, grey to dark brown, axial stripe dividing even broader, yellowish, paramedian stripes extending laterad down to reddish paraterga, below which sides yellow to yellow-brownish. Head, antennae (except for a whitish tip, including antennomere 7) and collum red-brown; venter, telson and basal podomeres whitish to light yellowish, legs growing increasingly brown distad.

Antennomere 6 only barely longer, but significantly higher than each of $2^{\text {nd }}$ and $3^{\text {rd}}$; antennae rather short, clavate, barely reaching only behind segment 2 when stretched dorsally $\left(\sigma^{7}\right)$. Tegument generally smooth and shining. In width, segments 2 and $3<$ head $<$ collum $<4<5-17$, thereafter body gently tapering towards telson. Head densely setose throughout. Lateral edges of collum broadly rounded, calluses being very thin. Paraterga (Figs 1-5) modestly developed, behind collum mostly bulb- or keel-shaped, set low (at about half of metazonital height), thinner in poreless, thicker in pore-bearing, segments, increasingly well produced beak-shaped behind tergal margin in all postcollum segments, clearly pointed, peritremata delimited by a complete and deep sulcus only dorsally, ventral sulcus being vague, arcuated, developed only in posterior quarter to $1 / 5^{\text {th }}$ extent. Lateral edge of paraterga devoid of indentations. Metaterga faintly rugulose at most. Collum and all following metaterga, including calluses, densely and irregularly hirsute, tergal setae being rather long, about 1/3-1/2 of metatergal length. Sulcus starting from segment 5 , deep, reaching the bases of paraterga. Stricture between pro- and metazona clearly beaded down to paraterga. Ozopores lying a little in front of caudal end of paraterga in a shallow ovoid groove, lateral, invisible from above. Pleurosternal carinae missing. Axial line sometimes barely traceable only in postsulcus halves of metaterga. Epiproct (Fig. 6) subtruncate, pre-apical lateral papillae small. Hypoproct (Fig. 7) semi-circular. Sterna unmodified. Sternal lobe between coxae 4 high, linguiform, densely setose (Fig. 8). Legs rather short and slender, about $1.2-1.3$ times times as long as midbody height $\left(\sigma^{7}\right)$; $\sigma^{7}$ tarsal brushes present until about legs 9 or 10, thereafter thinning out.

Gonopods rather simple (Figs 9-11). Coxite long, subcylindrical, with several distoventral setae; prefemoral portion small, about half as long as femorite, the latter slender, hollow on dorsal face, apicolateral lobe (I) small, rounded, dentiform. Solenophore long, about as long as femorite, clearly coiled, deeply bifid.

\section{Eustrongylosoma vanuatu sp.n.} Figs 12-20.

HOLOTYPE O' (MNHN JA 134), Vanuatu, Espiritu Santo Island, Funafus, Cave Tarius, inside cave, hand collection, $167.0201167^{\circ} \mathrm{E}, 15.53066667^{\circ} \mathrm{S}, 250 \mathrm{~m}$ a.s.1., 19.09.2006, leg. F. Brehier, S. Eberhard \& N. Lasson (SK06-19-04). 

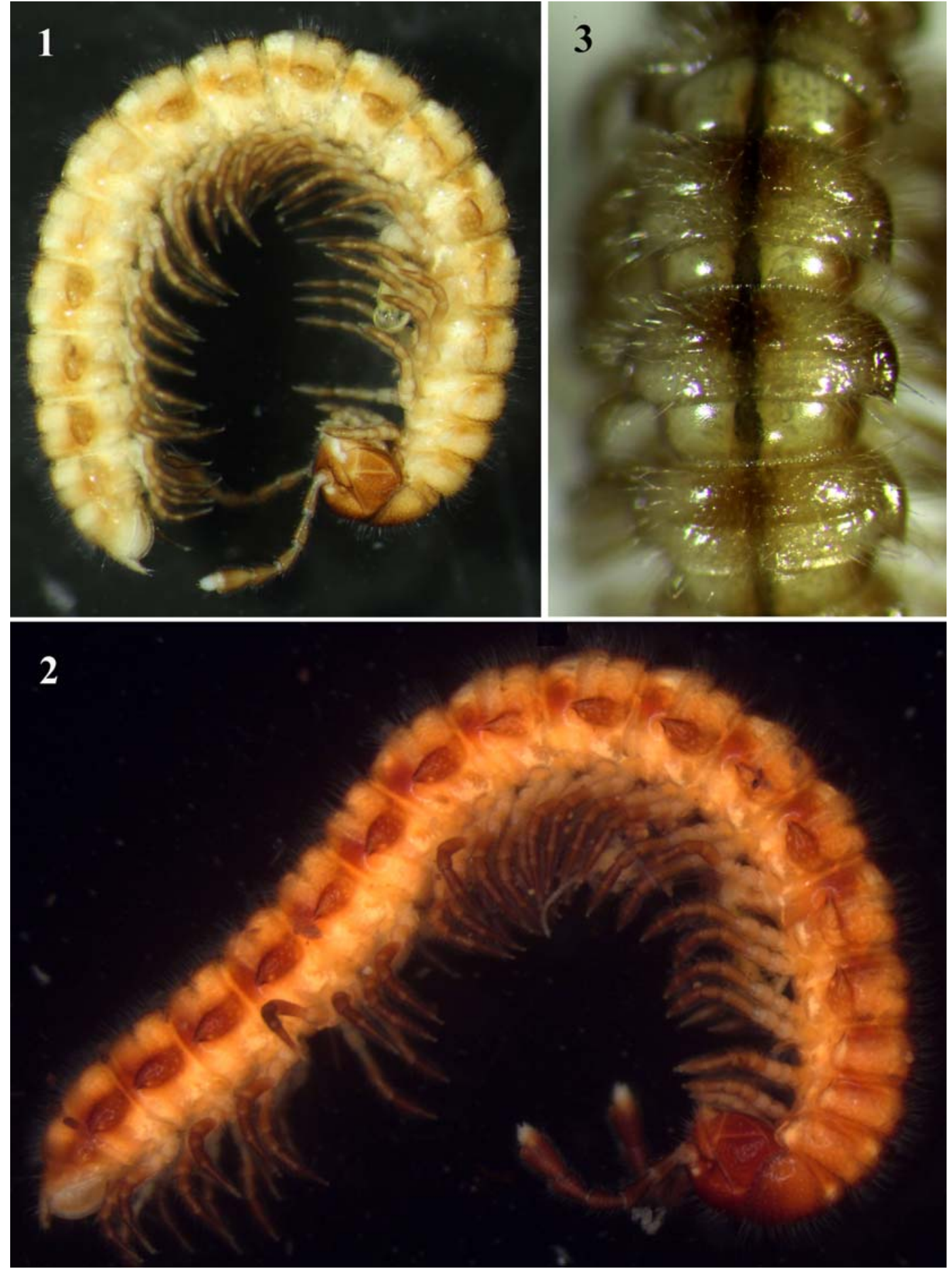

Figs 1-3. Eustrongylosoma hirsutum sp.n., $0^{7}$ holotype $\left(1 \&\right.$ 3) \& $0^{7}$ paratype (2): 1-2 - habitus, lateral view; 3 - midbody segments, dorsal view. Photographed not to scale.

Рис. 1-3. Eustrongylosoma hirsutum sp.n., голотип (1 и 3) и паратип О7 (2): 1-2 - внешний вид, сбоку; 3 - среднетуловищные сегменты, сверху. Сфотографировано без масштаба.

PARATYPES: $1 \sigma^{7}$ (MNHN JA 134), same locality, together with holotype; $1 \mathrm{O}^{7}$ (ZMUM), same locality, Funafus, Cave Kafae, hand collection, $167.0143333^{\circ} \mathrm{E}, 15.537^{\circ} \mathrm{S}, 18.09 .2006$, leg. S. Eberhard (SK06-18-10); 1 O (MNHN JA 134), 1 O (ZMUC), same locality, Funafus, Cave Riorua, hand collection, $167.0216^{\circ} \mathrm{E}$, $15.53578333^{\circ} \mathrm{S}, 250 \mathrm{~m}$ a.s.1., 18.09.2006, leg. C. Rahmadi (SK0618-12).
NAME. To emphasize the terra typica, a noun in apposition.

DIAGNOSIS. This new species is distinguished among congeners by the following combination of characters: a smooth and shining tegument; quite long antennae and legs; broad calluses on rather well devel- 


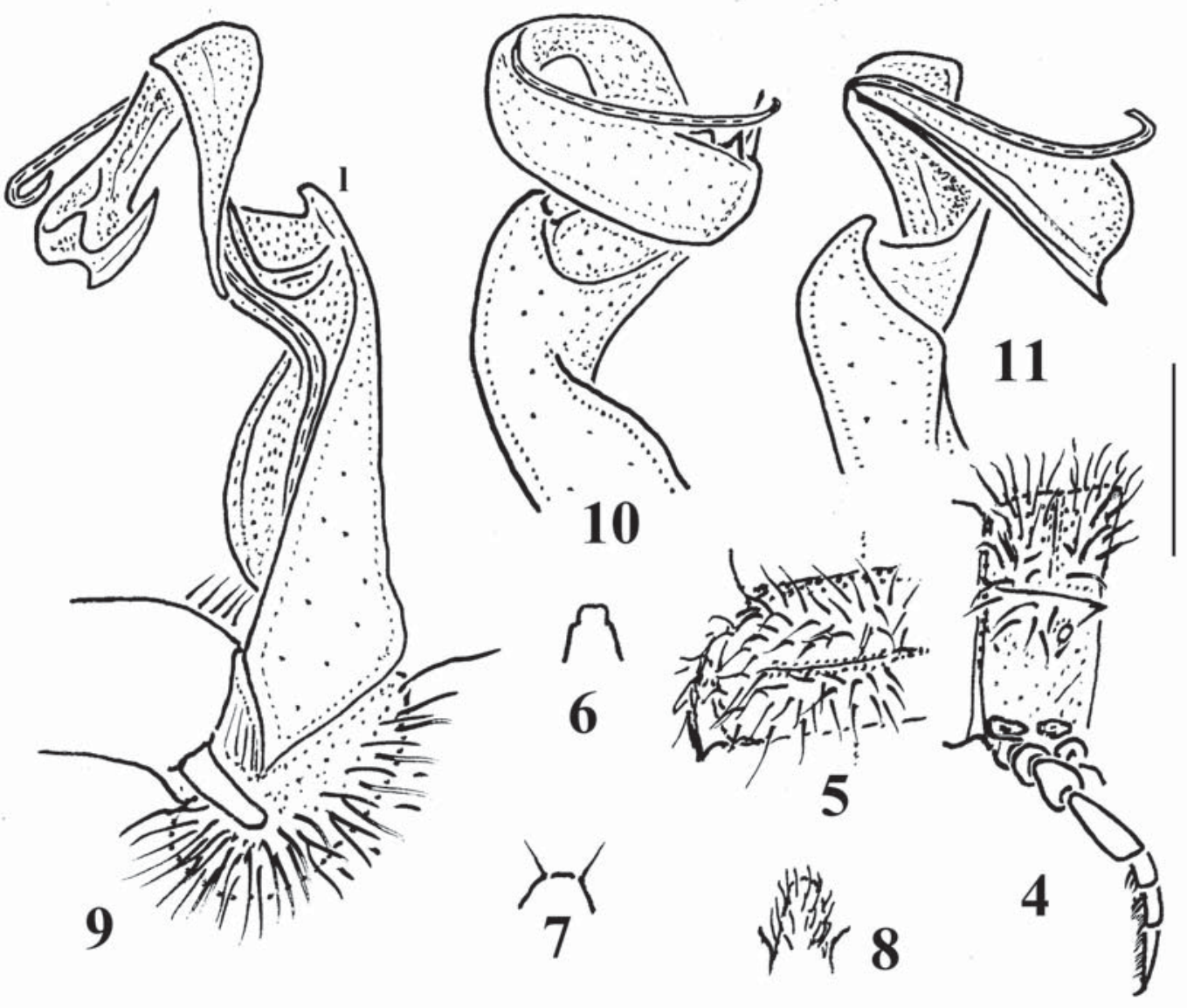

Figs 4-11. Eustrongylosoma hirsutum sp.n., $0^{7}$ paratype: 4-5 - segment 10, lateral and dorsal views, respectively; 6 - epiproct, dorsal; 7 - hypoproct, ventral; 8 - sternal lobe between coxae 4, caudoventral; 9-11 - left gonopod, mesal, ventral and lateral views, respectively. Scale bar: $0.5(4-8) \& 0.25 \mathrm{~mm}(9-11)$.

Рис 4-11. Eustrongylosoma hirsutum sp.n., паратип О7: 4-5 - сегмент 10, соответственно сбоку и сверху; 6 - эпипрокт, сверху; 7 - гипопрокт, снизу; 8 - стернальная пластина между тазиками 4, одновременно сзади и снизу; 9-11 - левый гонопод, соответственно изнутри, снизу и сбоку. Масштаб: 0,5 (4-8) и 0,25 мм (9-11).

oped, large and laterally rounded paraterga; always rounded caudal corners of paraterga never extending behind rear tergal margin; missing tergal setae (at least no setation pattern being traceable); a very high and linguiform lobe between $\sigma^{7}$ coxae 4; and an only poorly bifid solenophore devoid of any other evident structures.

DESCRIPTION. Length 15-20 mm, width of midbody pro- and metazona $1.0-1.1$ and $1.4-1.6 \mathrm{~mm}$, respectively $\left(\sigma^{7}\right)$. Holotype ca $15 \mathrm{~mm}$, width of pro- and metazona 1.0 and $1.4 \mathrm{~mm}$, respectively. Coloration uniformly light brown (Fig. 12), front body third sometimes slightly more intensely coloured, light brown, only genae, venter and legs lighter grey-yellowish to nearly pallid. Antennomeres 7 and 8 whitish.

Body submoniliform. Antennomeres 2 to 6 subequal in length, but $6^{\text {th }}$ highest; antennae long and slender, reaching behind segment 3 when stretched dorsally $\left(\sigma^{7}\right)$. Tegument generally smooth and shining. In width, head $=$ segments $6-17>$ segment $5>$ collum $=4$ $>2$ and 3 ; after segment 17 , body gently tapering towards telson. Head rather densely setose on clypeus and frons, but only a few setae on vertex. Collum semilunar; lateral edges broadly rounded. Paraterga (Figs 12-14) rather well developed, keel-shaped, subhorizontal, set low (at about 1/2-1/3 of metazonital height), only slightly thinner in poreless, thicker in pore-bearing, segments, never reaching behind tergal margin, barely touching the rear contour like rather narrowly rounded teeth only in segments 17-19, mostly quite broadly rounded caudally, delimited by a complete and deep sulcus only dorsally (yet nearly obliterate anteriorly), ventral sulcus being incomplete, developed only in posterior quarter to $1 / 5^{\text {th }}$ extent. Calluses wide, broadly convex laterally. Metaterga generally glabrous, faintly rugulose at most, a little more clearly so near transverse sulcus, sometimes with traces of an axial line, surface below paraterga usually very finely microgranular and rugulose. Tergal setae fully abraded, pattern untraceable. Sulcus starting from segment 2, a medially faintly impressed line in segments $2-4$ and 17 , a deep sulcus reaching the bases of paraterga in segments 5-16. Stricture between pro- and metazona nearly smooth, only finely striate down to paraterga. Ozo- 


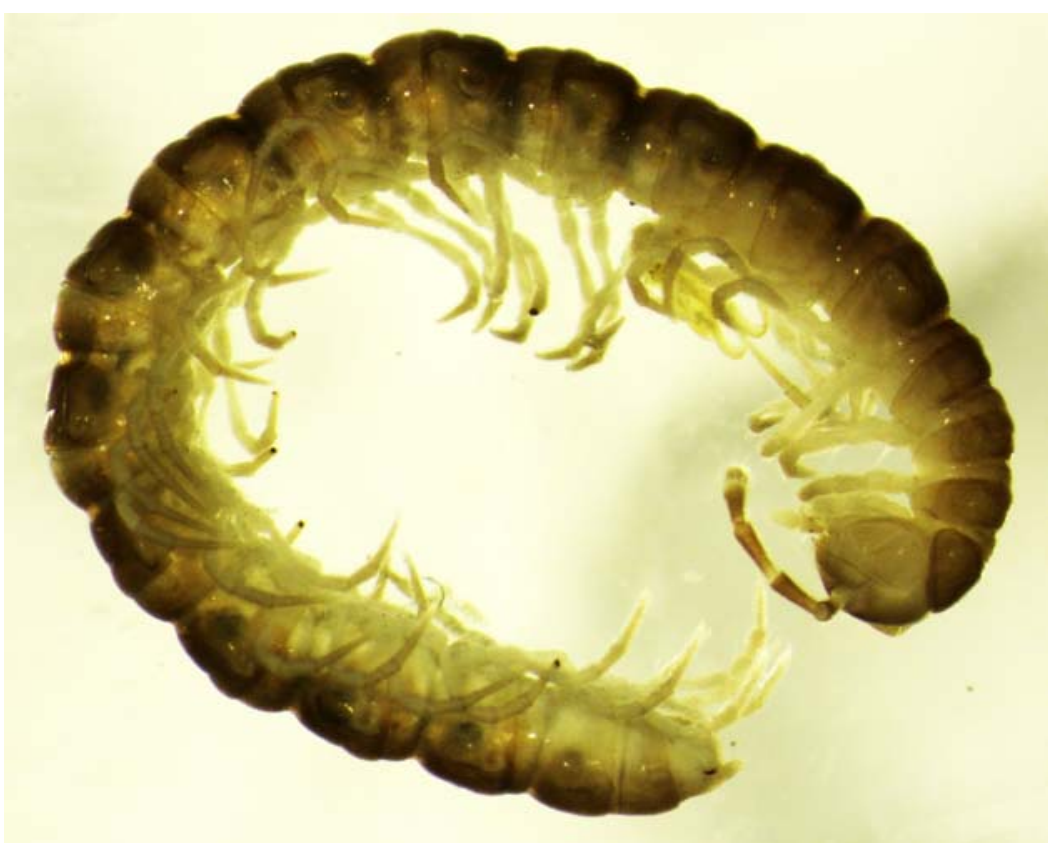

Fig. 12. Habitus of Eustrongylosoma vanuatu sp.n., O $^{\text {x }}$ paratype from Cave Kafae, lateral view. Photographed not to scale.

Рис. 12. Внешний вид Eustrongylosoma vanuatu sp.n., паратип О’ из пещеры Kafae, сбоку. Сфотографировано без масштаба.

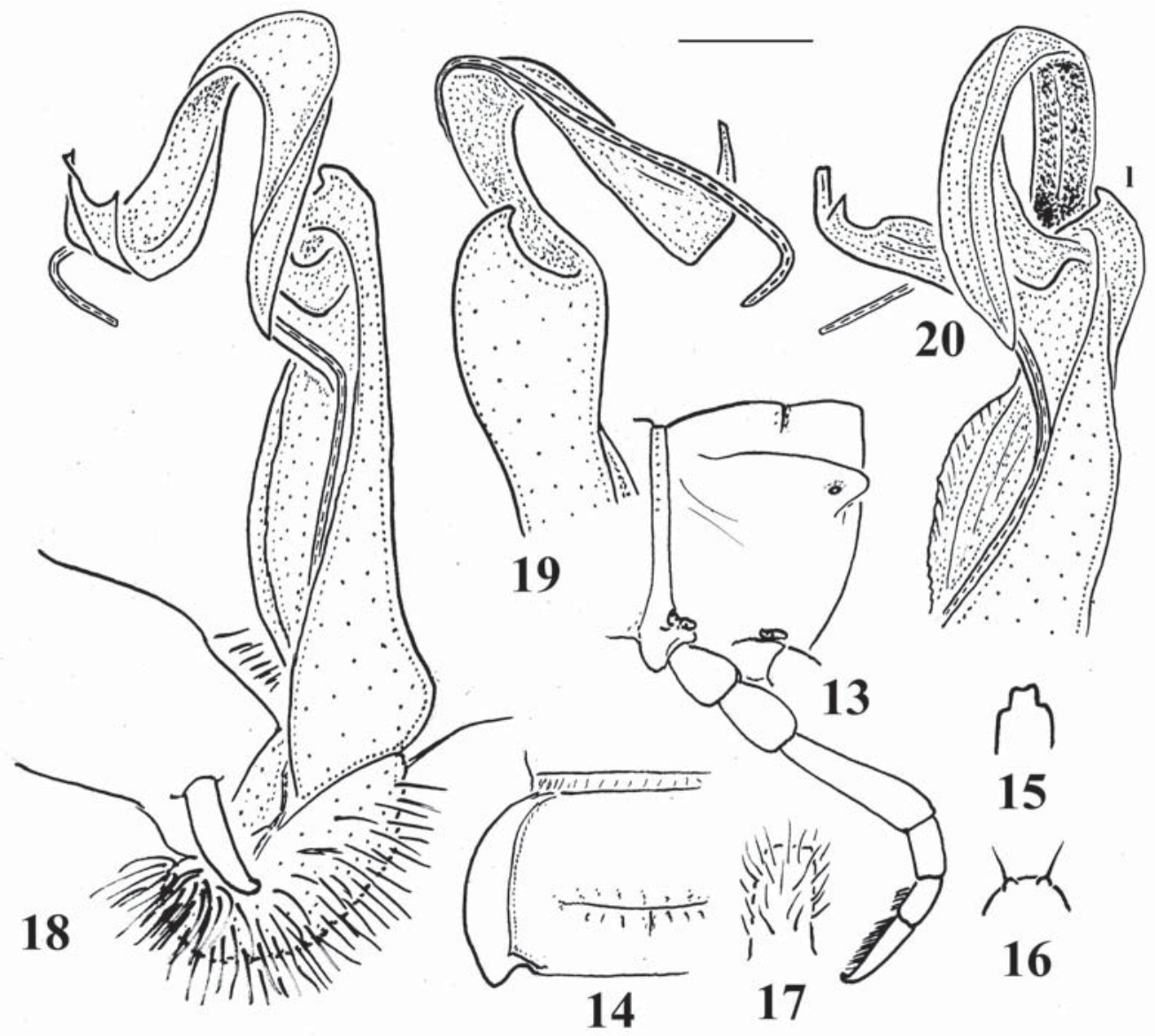

Figs 13-20. Eustrongylosoma vanuatu sp.n., O paratype: 13-14 - segment 10, lateral and dorsal views, respectively; 15 - epiproct, dorsal; 16 - hypoproct, ventral; 17 - sternal lobe between coxae 4, caudoventral; $18-20$ - left gonopod, mesal, ventral and lateral views, respectively. Scale bar: $0.5(13-17) \& 0.25 \mathrm{~mm}(18-20)$

Рис 13-20. Eustrongylosoma vanuatu sp.n., паратип О : 13-14 - сегмент 10, соответственно сбоку и сверху; 15 - эпипрокт, сверху; 16 - гипопрокт, снизу; 17 - стернальная пластина между тазиками 4, одновременно сзади и снизу; 18-20 - левый гонопод, соответственно изнутри, снизу и сбоку. Масштаб: 0,5 (13-17) и 0,25 мм (18-20). 


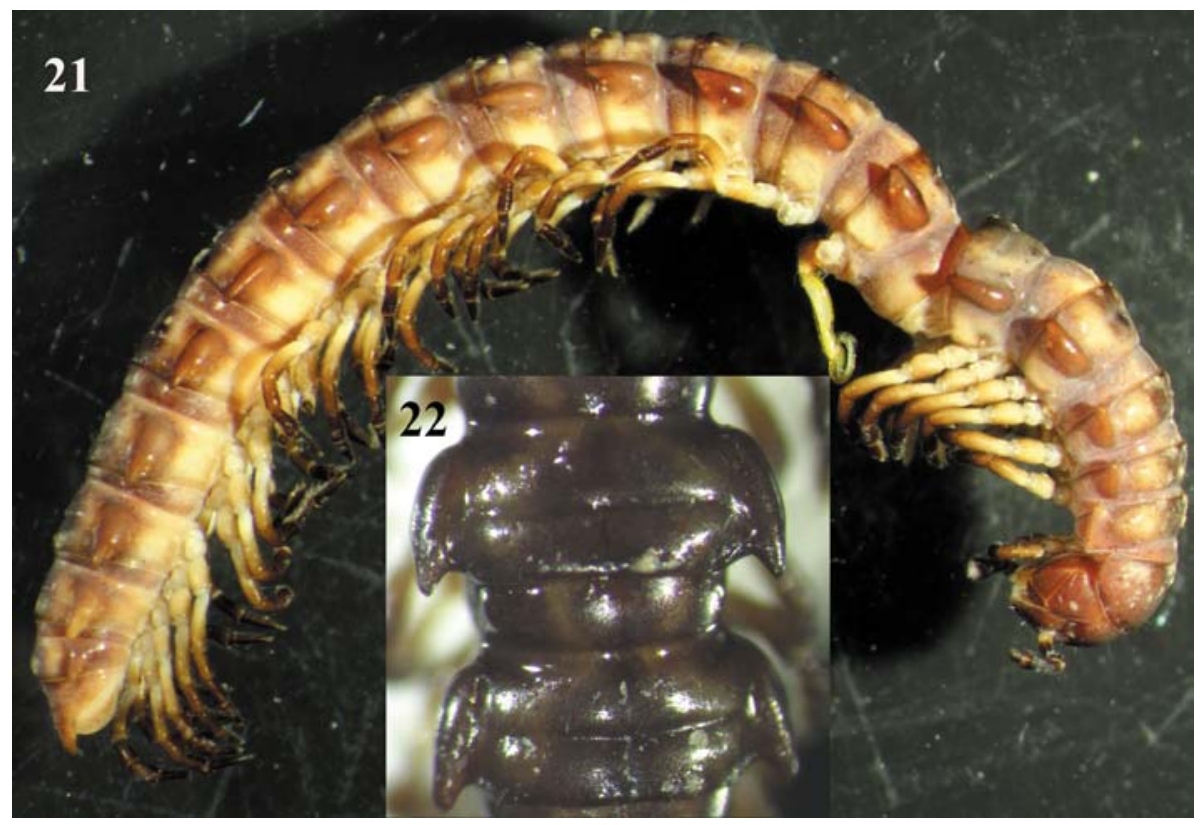

Figs 21-22. Eustrongylosoma acutum sp.n., holotype: 21 - habitus, lateral view; 22 - midbody segments, dorsal view. Photographed not to scale.

Рис. 21-22. Eustrongylosoma acutum sp.n., голотип: 21 - внешний вид, сбоку; 22 - среднетуловищные сегменты, сверху. Сфотографировано без масштаба.

pores lying close to caudal end of paraterga in a shallow ovoid groove, lateral, invisible from above. Pleurosternal carinae missing. Epiproct (Fig. 15) subtruncate, pre-apical lateral papillae rather large. Hypoproct (Fig. 16) semi-circular, caudal 1+1 setae strongly separated, borne on minute knobs. Sterna unmodified. Sternal lobe between coxae 4 linguiform, high, densely setose (Fig. 17), bulged caudally in basal half. Legs very long and slender, ca 2.0 times as long as midbody height $\left(\sigma^{7}\right)$; $\sigma^{7}$ tarsal brushes traceable until about legs of segment 15 or 16 , thereafter gradually thinning out.

Gonopods rather simple (Figs 18-20). Coxite long, subcylindrical, setose distoventrally; prefemoral portion rather small, about half as long as femorite, the latter slender, apicolateral lobe (l) evident, dentiform, slightly curved at apex. Solenophore long, coiled, rather faintly bifid, both ends being subequal and dentiform.

\section{Eustrongylosoma acutum sp.n.} Figs 21-29.

HOLOTYPE O' (MNHN JA 135), Vanuatu, Espiritu Santo Island, Pao Forest, $166.66668^{\circ} \mathrm{E}, 14.9434^{\circ} \mathrm{S}, 710 \mathrm{~m}$ a.s.l., forest litter, 20.11.2006, leg. C. Rollard (S06-20-R).

PARATYPE: $1 \mathrm{O}^{7}$ (MNHN JA 135), same locality, date and collector unknown (Po64).

NAME. To emphasize that most of the paraterga are acute caudally.

DIAGNOSIS. This new species is distinguished among congeners by the following combination of characters: a smooth and shining tegument; quite long antennae and legs; most of paraterga with acute caudal corners well protruding behind rear tergal margin; metaterga with three transverse rows of $3+3$ setae; transverse sulcus complete on metaterga 5-17, incomplete on $18^{\text {th }}$; mediocre calluses on rather high and well-developed paraterga; a subtrapeziform lobe between $\sigma^{7}$ coxae 4 ; and a broadly subtruncate and poorly undulate solenophore apex.

DESCRIPTION. Length ca 19 (paratype) or $21 \mathrm{~mm}$ (holotype), width of midbody pro- and metazona 1.3 and 1.8 (paratype) or 1.5 and $2.0 \mathrm{~mm}$ (holotype), respectively. Coloration rather uniformly castaneous brown, head entirely infuscate, pattern traceable due to two vague, rather light grey- to yellow-brown, narrow, paramedian stripes covering bases of paraterga and extending onto prozona, and similarly light grey- to yellow-brown sides, venter and $2-3$ basalmost podomeres (Fig. 21). Tips of antennae whitish.

Antennomeres 2 to 6 subequal in length, antennae rather short, reaching behind body segment 2 when stretched dorsally $\left(\sigma^{7}\right)$. Tegument generally smooth and shining. In width, segments 2 and $3<$ collum $=4<$ head $=$ segments $5(6)-17$, thereafter body gently tapering towards telson. Head densely setose on clypeus and frons, only with a few setae on vertex. Collum semilunar, bearing three transverse rows of $4+4$, rather long setae in front row, and $2+2$ and $2+2$ in both following rows; lateral edges broadly rounded. Paraterga (Figs 21-24) rather well developed, keel-shaped, subhorizontal, set rather high (at about $1 / 3^{\text {rd }}$ of metazonital height), slightly thinner in poreless, thicker in porebearing, segments, strongly produced beak-shaped behind tergal margin in segment 2 , less so, but increasingly strongly until $18^{\text {th }}$, only slightly shorter in $19^{\text {th }}$, caudal corner mostly pointed or nearly so, frontolateral 

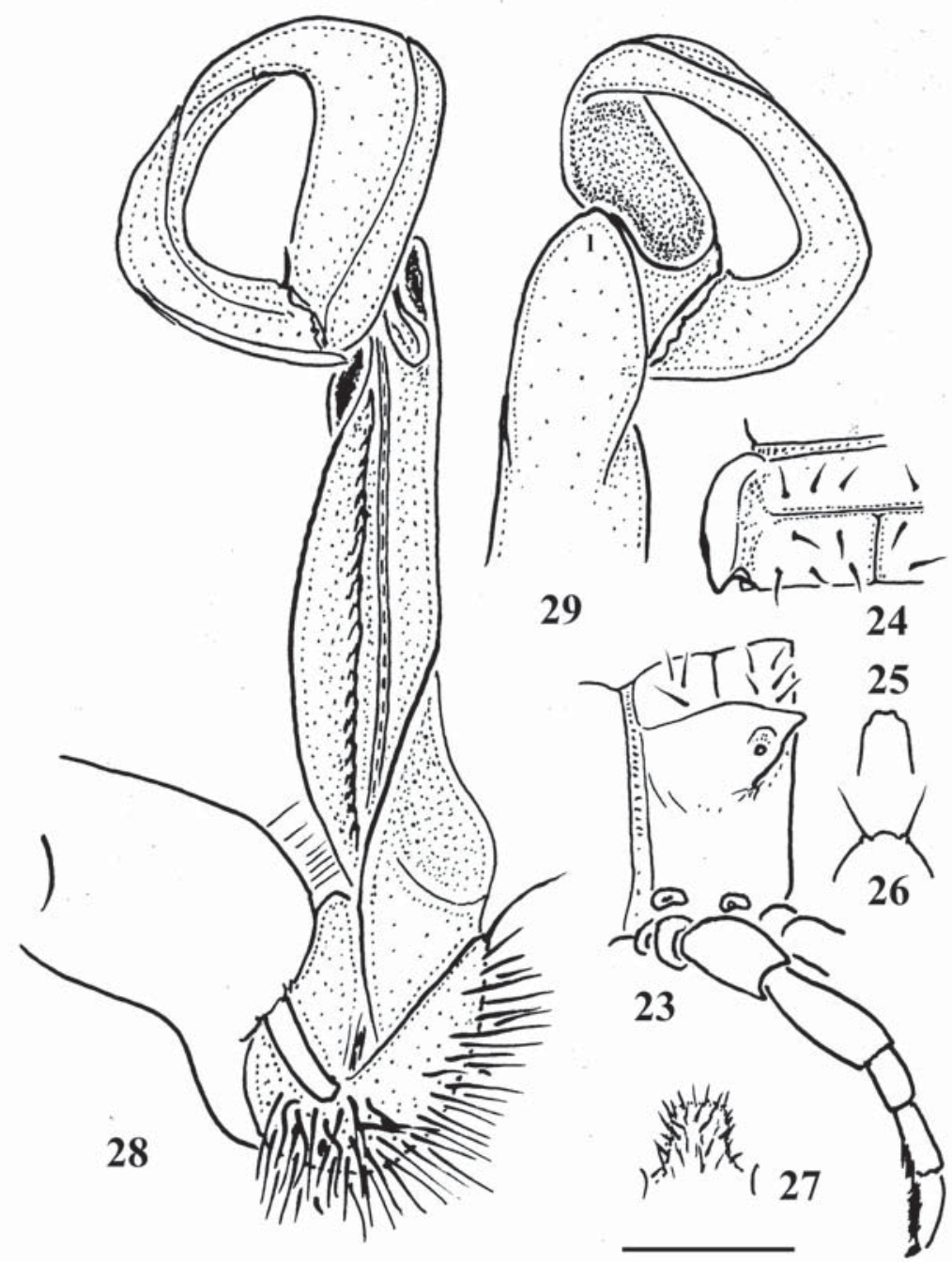

Figs 23-29. Eustrongylosoma acutum sp.n., holotype: $23-24$ - segment 10, lateral and dorsal views, respectively; 25 - epiproct, dorsal; 26 - hypoproct, ventral; 27 - sternal lobe between coxae 4, caudoventral; 28-29 - left gonopod, mesal and lateral views, respectively. Scale bar: 1.0 (23-27) \& $0.25 \mathrm{~mm}(28-29)$.

Рис 23-29. Eustrongylosoma acutum sp.n., голотип: 23-24 - сегмент 10, соответственно сбоку и сверху; 25 - эпипрокт, сверху; 26 гипопрокт, снизу; 27 - стернальная пластина между тазиками 4, одновременно сзади и снизу; 28-29 - левый гонопод, соответственно изнутри и сбоку. Масштаб: 1,0 (2327) и 0,25 мм (28-29).

Fig. 30. Habitus of Eustrongylosoma picturatum sp.n., holotype, lateral view. Photographed not to scale.

Рис. 30. Внешний вид Eustrongylosoma picturatum sp.n., голотип, сбоку. Сфотографировано без масштаба.

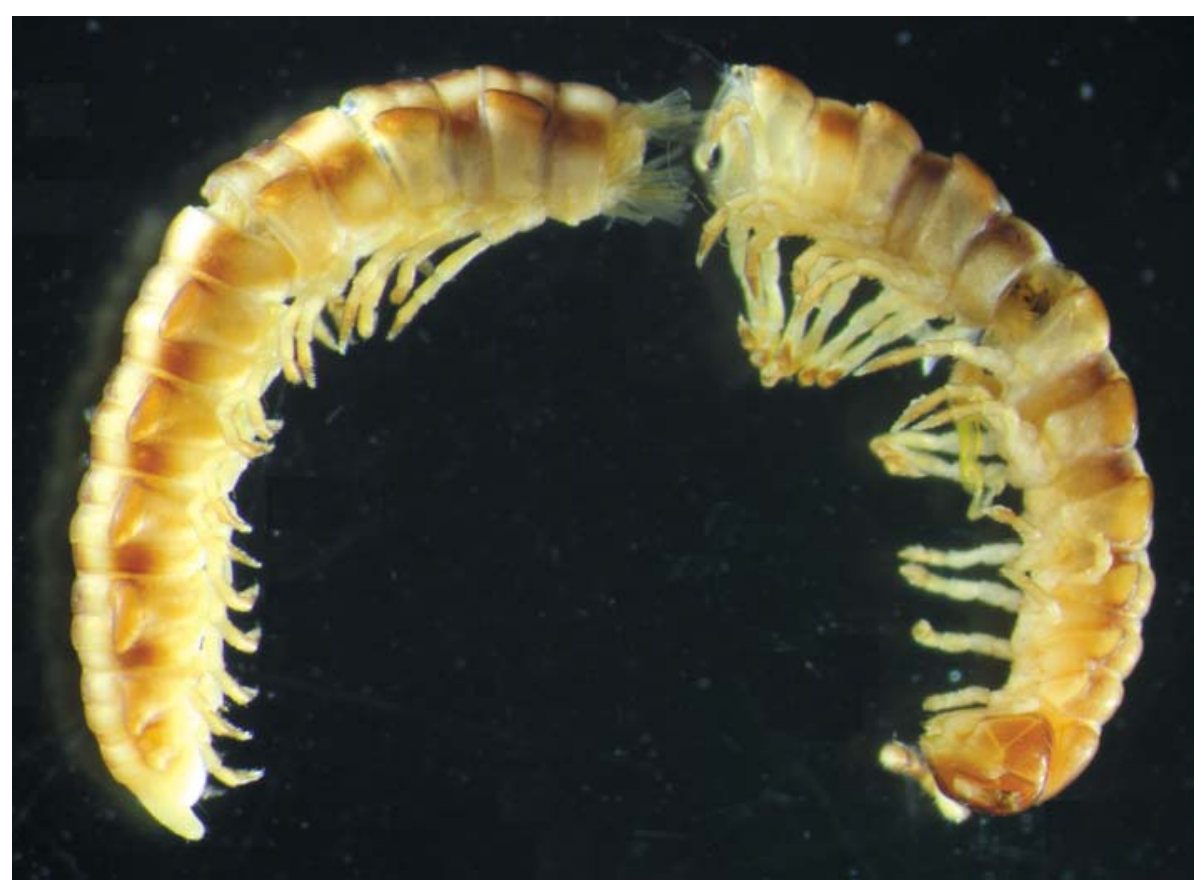



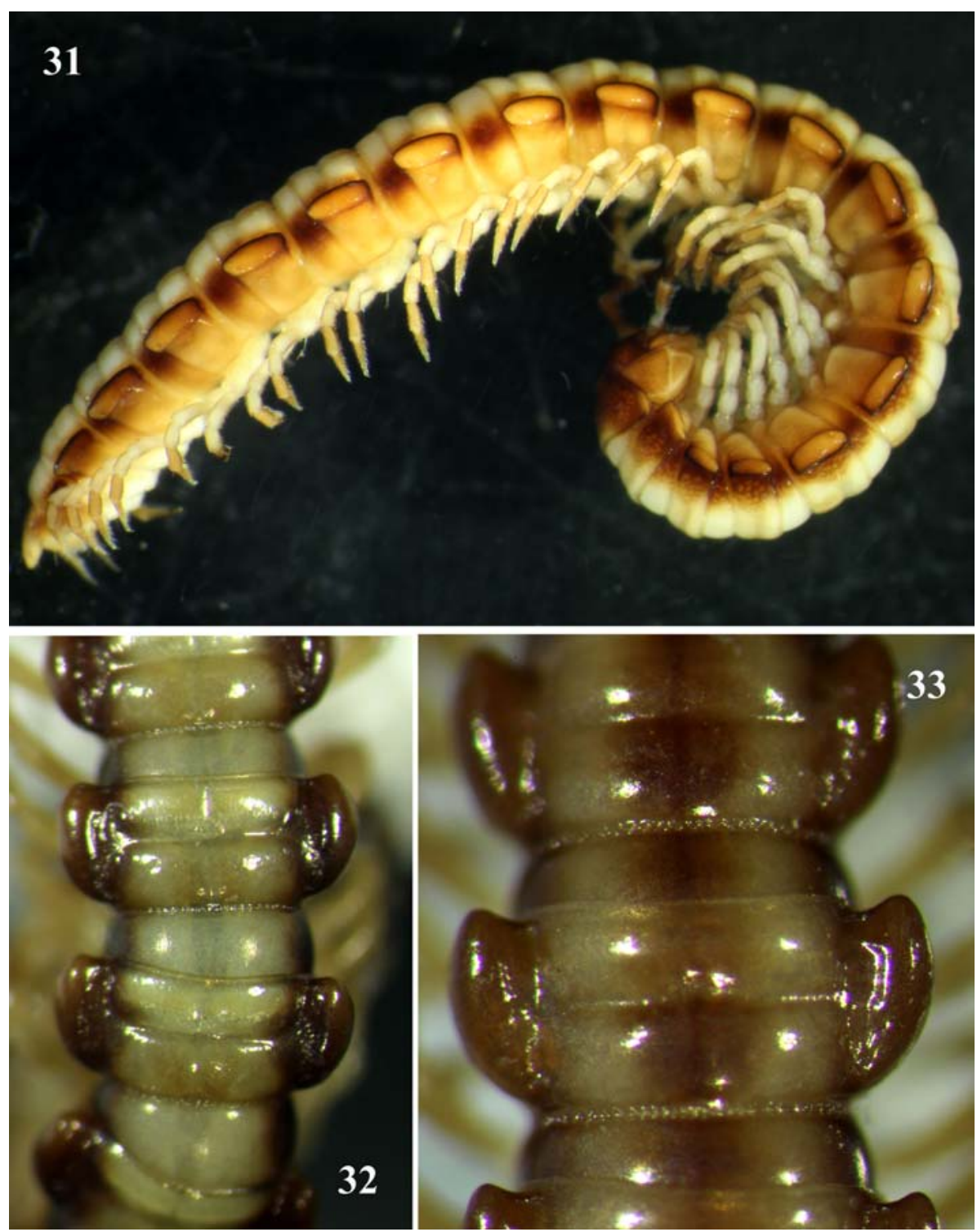

Fig. 31-33. Eustrongylosoma picturatum sp.n., $0^{7}$ paratype from Cave Fapon: 31 - habitus, lateral view; 32-33 - midbody segments, dorsal view. Photographed not to scale.

Pис. 31-33. Eustrongylosoma picturatum sp.n., паратип О7 из пещеры Fapon: 31 - внешний вид, сбоку; 32-33 среднетуловищные сегменты, сверху. Сфотографировано без масштаба.

shoulder and lateral margin broadly rounded, calluses rather wide, delimited by a complete and deep sulcus only dorsally, ventral sulcus being incomplete, developed only in posterior quarter to $1 / 5^{\text {th }}$ extent. Metaterga smooth, only in places faintly rugulose, a little more clearly so near sulcus, surface below paraterga very finely rugulose. Tergal setae rather long, about $1 / 5^{\text {th }}$ of metatergal length, mostly arranged in three rows of $3+3$ in each, one in front of, both others behind, sulcus, pattern usually well traceable as insertion points or, especially in caudal row, minute knobs (Figs 23 \& 24). Sulcus starting from segment 5, rather deep, complete, reaching the bases of paraterga until segment 17 , abbreviated and more superficial in $18^{\text {th }}$, absent from $19^{\text {th }}$. Stricture between pro- and metazona finely and dense- ly striolate down to paraterga. Ozopores lying close to caudal end of paraterga in a shallow ovoid groove, lateral, invisible from above. Pleurosternal carinae nearly wanting, very poorly developed ridges only in segment 2 , low rounded bulges until segment 7 , thereafter totally suppressed $\left(O^{7}\right)$. Axial line evident only in postsulcus halves of metaterga, more rarely barely traceable in front halves as well. Epiproct (Fig. 25) subtruncate, pre-apical lateral papillae very small. Hypoproct (Fig. 26) semi-circular, caudal 1+1 setae well separated, borne on minute knobs. Sterna unmodified. Sternal lobe between coxae 4 subtrapeziform, densely setose (Fig. 27). Legs long and slender, about 1.7-1.9 times as long as midbody height $\left(\sigma^{7}\right)$; $\sigma^{7}$ distotibial and tarsal brushes present until last two leg-pairs. 


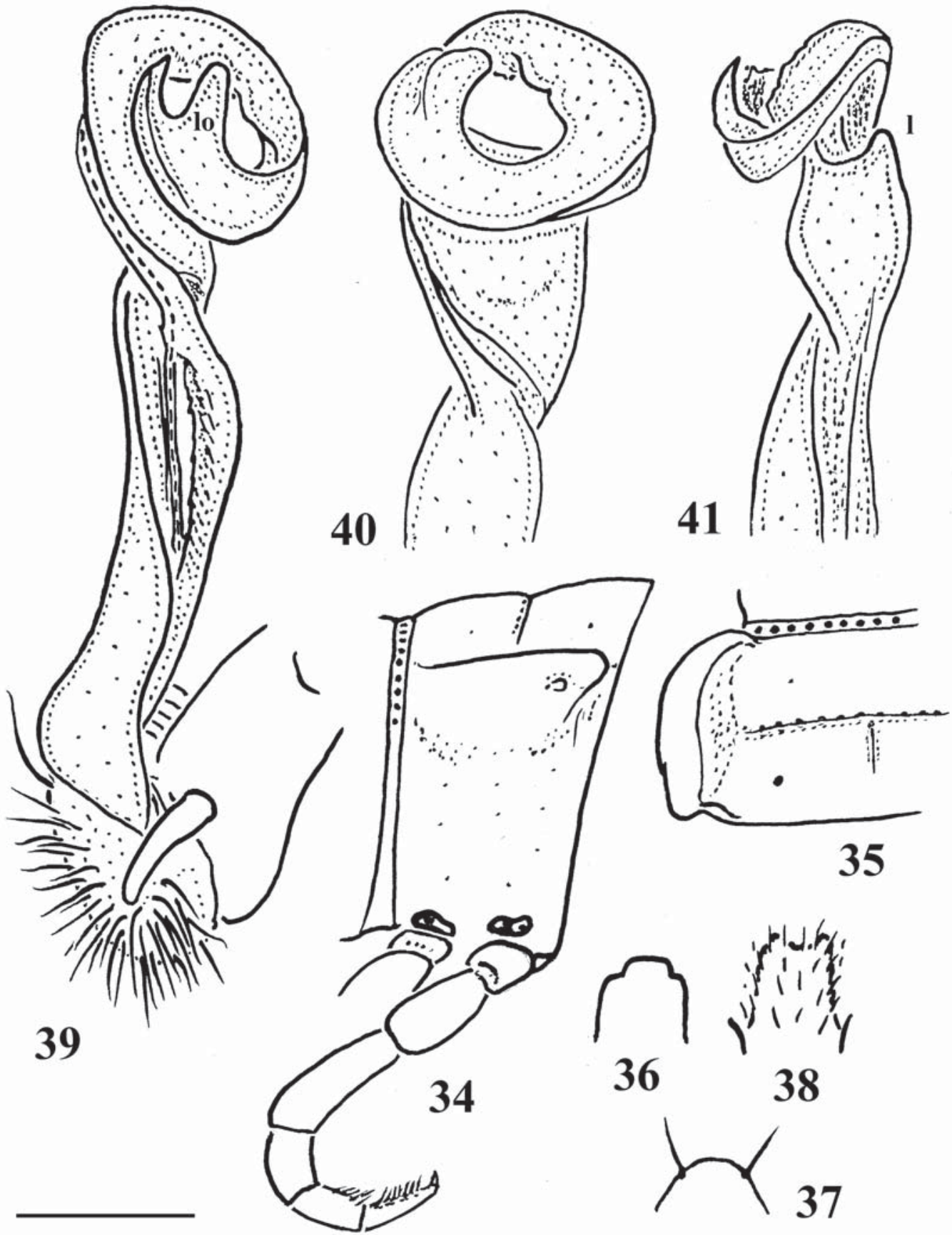

Figs 34-41. Eustrongylosoma picturatum sp.n., holotype: 34-35 - segment 10, lateral and dorsal views, respectively; 36 - epiproct, dorsal; 37 - hypoproct, ventral; 38 - sternal lobe between coxae 4, caudoventral; 39-41 - right gonopod, mesal, dorsal and lateral views, respectively. Scale bar: 1.0 (34-38) \& $0.25 \mathrm{~mm}(39-41)$.

Рис 34-41. Eustrongylosoma picturatum sp.n., голотип: 34-35 - сегмент 10, соответственно сбоку и сверху; 36 - эпипрокт, сверху; 37 - гипопрокт, снизу; 38 - стернальная пластина между тазиками 4, одновременно сзади и снизу; 39-41 - правый гонопод, соответственно изнутри, сверху и сбоку. Масштаб: 1,0 (34-38) и 0,25 мм (39-41).

Gonopods simple (Figs 28 \& 29). Coxite long, subcylindrical, with several setae distoventrally; prefemoral portion small, about $1 / 3^{\text {rd }}$ as long as femorite, the latter slender, ventral lobe serrate at margin and somewhat better developed than dorsal one, apicolateral lobe (l) rounded and simple. Solenophore subcircular, devoid of any outgrowths, with a broadly truncate and slightly undulate tip.

\section{Eustrongylosoma picturatum sp.n.}

Figs 30-49.

HOLOTYPE O (MNHN JA 136), Vanuatu, Espiritu Santo Island, Pao Forest, $166.66668^{\circ} \mathrm{E}, 14.9434^{\circ} \mathrm{S}, 710 \mathrm{~m}$ a.s.1., forest litter, 20.11.2006, leg. C. Rollard (S06-20-R).

PARATYPES: $10^{7}$ (MNHN JA 136), same locality, Boutmas, Cave Fapon, hand collection, $166.9648833^{\circ} \mathrm{E}, 15.33101667^{\circ} \mathrm{S}$, 12.08.2005, leg. J. Lips (SK05-Lips23); 1 o (ZMUM), 1 \% 


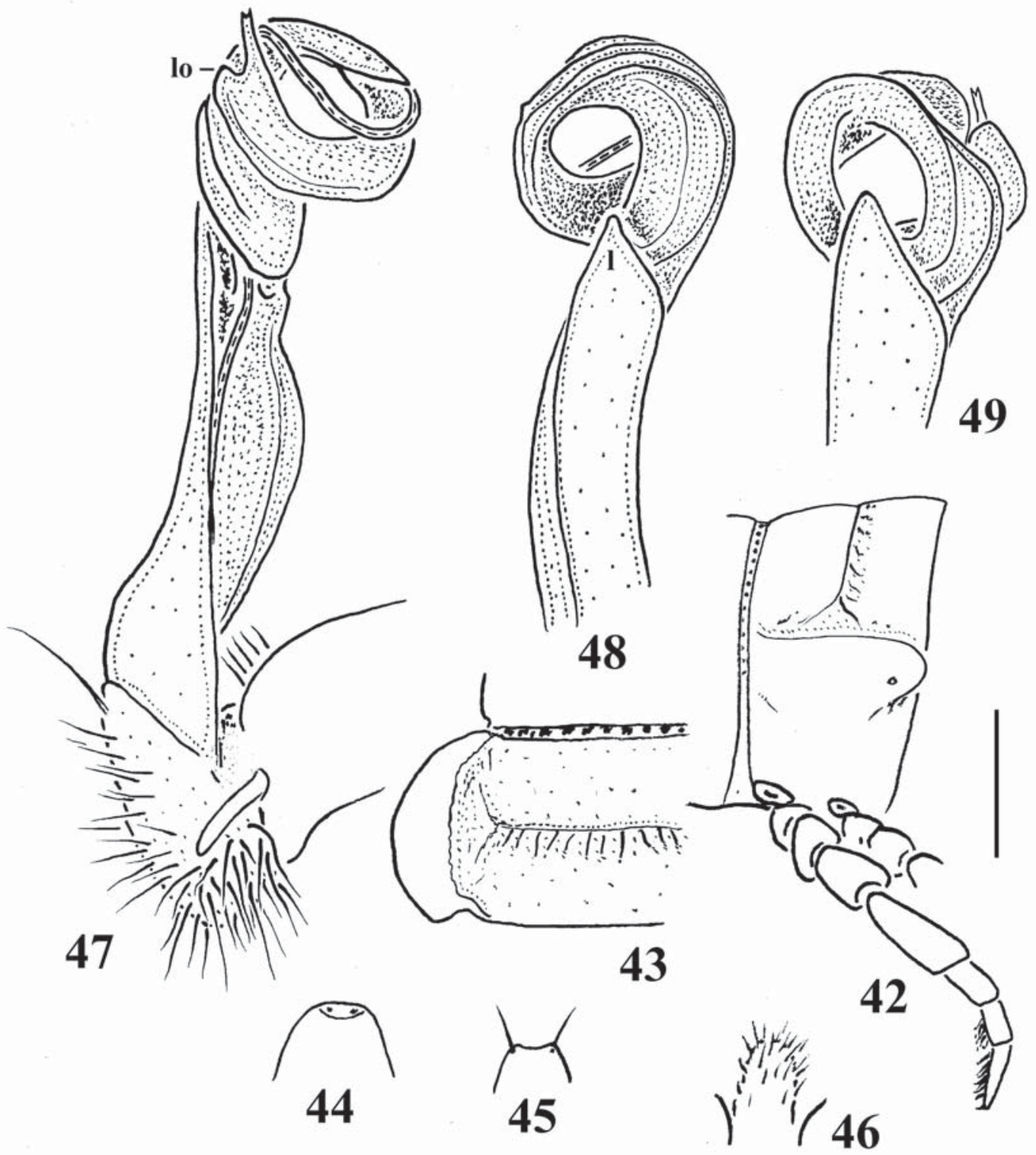

Figs 42-49. Eustrongylosoma picturatum sp.n., $\mathrm{O}^{7}$ paratype from Cave Fapon: $42-43$ - segment 10, lateral and dorsal views, respectively; 44 - epiproct, dorsal; 45 - hypoproct, ventral; 46 - sternal lobe between coxae 4, caudoventral; 47-49 — right gonopod, mesal, ventral and lateral views, respectively. Scale bar: 0.5 (42-46) \& $0.25 \mathrm{~mm}(47-49)$.

Рис 42-49. Eustrongylosoma picturatum sp.n., паратип $0^{7}$ из пещеры Fapon: 42-43 - сегмент 10, соответственно сбоку и сверху; 44 - эпипрокт, сверху; 45 - гипопрокт, снизу; 46 - стернальная пластина между тазиками 4, одновременно сзади и снизу; 47-49 - правый гонопод, соответственно изнутри, снизу и сбоку. Масштаб: 0,5 (42-46) и 0,25 мм (47-49).

(ZMUC), 19,2 juv. (MNHN JA 136), same locality, Natawa, forest, litter, Berlese extraction after sifting, $167.183167^{\circ} \mathrm{E}, 15.2962^{\circ} \mathrm{S}$, 21.09.2006, leg. L. Deharveng \& A. Bedos (SK06-21-29).

NAME. To emphasize the characteristic, highly vivid colour pattern.

DIAGNOSIS. This new species is distinguished among congeners by its characteristic, mostly highly vivid colour pattern, coupled with well-developed, often very broad and laterally convex paraterga which are always rounded at the caudal corner, the latter mostly not extending behind the rear tergal margin, a simple, small, rounded apicolateral lobe of the gono- pod femorite, and a subterminal rounded lobule and a subacuminate tip of the solenophore.

DESCRIPTION. Length of adults $15-16 \mathrm{~mm}$, width of midbody pro- and metazona $0.9-1.0$ and $1.4-1.5$ $\left(O^{7}\right)$ or 1.1 and $1.5 \mathrm{~mm}(+)$, respectively. Holotype ca $16 \mathrm{~mm}$ long, 1.0 and $1.5 \mathrm{~mm}$ wide on midbody proand metazona, respectively. Coloration and pattern much like in E. acutum sp.n., but in alcohol pattern often even more vivid (Figs 30-33); dorsum either completely yellow-whitish $\left(\sigma^{7}\right.$ paratype from Cave Fapon) or divided into three subequally wide and bright longitudinal stripes, median one being dark brown to 
light brownish (holotype) and in places interrupted into individual spots, while both paramedian stripes yellowish to greyish and extending down to paraterga; a somewhat narrower, dark ( $\sigma^{7}$ paratype from Natawa) or light brownish to red-brown stripe (holotype \& some paratypes) covering paraterga (sometimes including their bases) and extending onto prozona; sides below paraterga and entire telson as light as both paramedian stripes (holotype) (Fig. 30). Legs and antennae light, slightly infuscate, brown to brownish only distad.

Antennomeres 2 to 6 subequal in length; antennae short, distinctly clavate, reaching only behind segment $2\left(\sigma^{7}\right)$ or collum $(+)$ when stretched dorsally. Tegument generally smooth and shining. In width, segment $3<$ collum $<$ segments 2 and $4<$ head $=5$ 17 , thereafter body gently tapering towards telson. Head densely setose on clypeus and frons, nearly bare on vertex. Collum semi-lunar, bearing at least two transverse rows of setae, one row of very short setae along front margin, second row caudally; setation pattern untraceable; lateral edges broadly rounded. Paraterga (Figs 30-35, 42 \& 43) rather well-developed, a little less so in + compared to $\sigma^{7}$, keel-shaped, subhorizontal, set rather high (at about $1 / 3^{\text {rd }}$ of metazonital height), a little lower in + compared to $\sigma^{7}$, only slightly thinner in poreless than in pore-bearing segments, slightly reaching behind tergal margin only in segments 2, 17 and 18, mostly just reaching the margin, caudal corner always rounded, more narrowly so only in segments 15-18; calluses medium-sized to broad, delimited by a complete and deep sulcus only dorsally, ventral sulcus being incomplete, developed only in posterior quarter to $1 / 5^{\text {th }}$ extent; frontolateral shoulders and lateral margin broadly rounded. Metaterga smooth, faintly rugulose at most, a little more clearly so near sulcus, surface below paraterga faintly rugulose. Tergal setae in postcollum segments minute, nearly fully or, more often, fully abraded, probably arranged in two transverse rows of $3+3$ or $4+4(?)$ in each, one lying in front of sulcus, second near caudal margin; setation pattern unclear. Sulcus starting from segment 2, superficial and incomplete in segments 2-4, deep, complete and almost or completely reaching the bases of paraterga in segments 517 , superficial and incomplete in $18^{\text {th }}$, absent from $19^{\text {th }}$. Stricture between pro- and metazona clearly beaded down to paraterga. Ozopores lying close to caudal end of paraterga in a shallow ovoid groove, lateral, invisible from above. Pleurosternal carinae nearly wanting, poorly developed bulges visible only in segments 2-7. Axial line in places visible, especially so in postsulcus halves of metaterga. Epiproct (Figs 36 $\& 44)$ subtruncate, pre-apical lateral papillae small to virtually missing. Hypoproct (Figs 37 \& 45) semicircular to subtrapeziform, caudal $1+1$ setae strongly separated. Sterna unmodified. Sternal lobe between coxae 4 nearly linguiform, densely setose, tip from emarginate to subtruncate (Figs 38 \& 46). Legs rather short, ca 1.4-1.5 $\left(\bigcirc^{7}\right)$ or 1.0-1.1 ( + ) times as long as

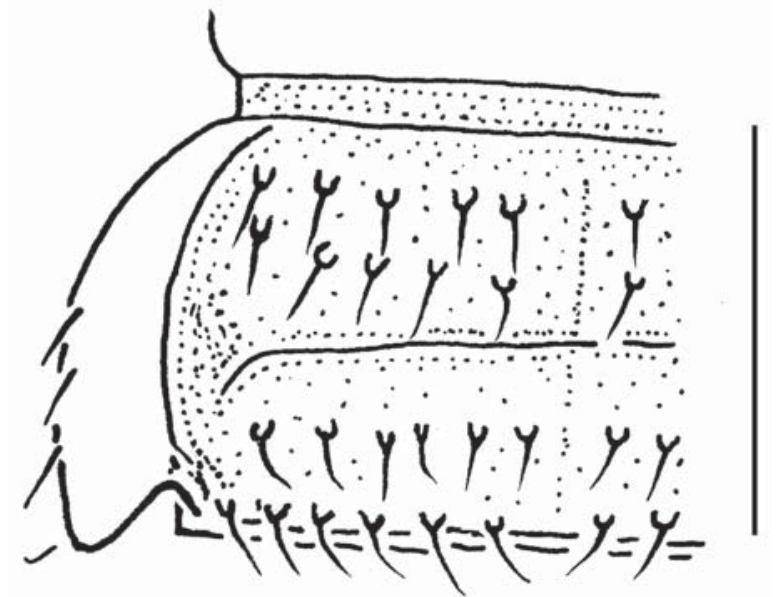

Fig. 50. Eustrongylosoma sp., + , segment 10, dorsal view. Scale: $1.0 \mathrm{~mm}$.

Рис. 50. Eustrongylosoma sp., + , сегмент 10, сверху. Масштаб: 1,0 мм.

midbody height; $\sigma^{\top}$ distotibial and tarsal brushes traceable until two last leg-pairs.

Gonopods rather simple (Figs 39-41, 47-49). Coxite long, subcylindrical, with several distoventral setae; prefemoral portion small, nearly $1 / 3^{\text {rd }}$ to about half as long as femorite, the latter slender, ventral lobe sometimes finely serrulate and somewhat better developed than dorsal one, apicolateral lobe (I) inconspicuous, small, dentiform, rounded. Solenophore subcircular, with a well-developed, rounded, subterminal lobule (lo) and a subacuminate tip.

\section{Eustrongylosoma sp.}

Fig. 50.

MATERIAL. 1 (MNHN JA 137), Vanuatu, Espiritu Santo Island, near Penaoru, summit of Mt Porotsits, $166.68103^{\circ} \mathrm{E}$, $14.96700^{\circ} \mathrm{S}, 1100 \mathrm{~m}$ a.s.1., forest litter, 17.11.2006, leg. C. Rollard (S06-17-BO)

REMARKS. This seems to be still another species of Eustrongylosoma in Vanuatu. It differs by a considerably larger size (length ca $25 \mathrm{~mm}$, width of midbody segments $2.8 \mathrm{~mm}$ ), uniform brown coloration (only legs and venter light brown), a special pattern of metatergal pilosity (two more or less regular rows of 5-6+5-6 short, acute, often curved setae on knobs both in front of and behind transverse sulcus until segment 14; from segment 15 on, postsulcus halves bearing 3-4 irregular rows of 6-8+6-8 such setae), and metatergal sulci fully developed and reaching bases of paraterga in segments $2-17$, nearly absent from $18^{\text {th }}$, fully absent from $19^{\text {th }}$

In the absence of male material, we refrain from naming this peculiar species. Superficially, it resembles the sympatric E. hirsutum sp.n. in the abundant metatergal pilosity, but differs clearly through the setae being considerably shorter and mostly arranged in four transverse, rather regular rows (Fig. 50). 


\section{Conclusion}

Above are only some of the species of the genus that actually populate the island, for numerous SANTO 2006 samples containing Eustrongylosoma material remain still untreated. Besides Eustrongylosoma, Espiritu Santo seems to fail to support any other presumed endemics from the family Paradoxosomatidae, but speciation in Eustrongylosoma in Vanuatu appears to be remarkable. Despite some encounters in caves, none of the species demonstrates obvious troglomorphic traits.

Based on the available evidence, the Vanuatu diplopod fauna is clearly depauperate, mainly being a derivative of that of New Guinea. Larger endemic Juliformia seem to have totally failed to populate Espiritu Santo, but the diversity of smaller species, especially from the order Polydesmida, is rather high, amounting to a few dozen.

ACKNOWLEDGEMENTS. We are most grateful to Louis Deharveng, Anne Bedos (both MNHN), Josiane Lips (Villeurbanne, France) and Christine Rollard (MNHN) for having rendered us their collections of Diplopoda for study. In addition, L. Deharveng and A. Bedos very helpfully took the pictures. Special thanks go to the MNHN administration for financing the first author's stay in Paris in March and April 2013.

\section{References}

Bouchet P., Le Guyader H., Pascal O. 2009. The SANTO 2006 Global Biodiversity Survey: An attempt to reconcile the pace of taxonomy and conservation // Zoosystema. Vol.31. No.3. P.401-406.

Bouchet P., Le Guyader H., Pascal O. (eds.). 2011. The Natural History of Santo // Patrimoines Naturels. Paris: Muséum national d'Histoire naturelle. No.70. 572 pp.

Bouchet P., Le Guyader H., Pascal O. 2012. The altruism of biodiversity exploration expeditions // Zoosystema. Vol.34. No.2. P.193-202.
Brolemann H.-W. 1931. Myriapodes recueillis par Madame Pruvot en Nouvelle-Calédonie et aux Loyalty // Archive de Zoologie expérimentale et générale. T.72. Fasc.2. P.275-316.

Golovatch S.I. 1997. On several new or poorly-known Oriental Paradoxosomatidae (Diplopoda, Polydesmida), V // Arthropoda Selecta. Vol.5. Nos 3/4. P.131-141 (for 1996).

Golovatch S.I., Geoffroy J.-J, Mauriès J.-P., VandenSpiegel D. 2008. The first, new species of the millipede family Pyrgodesmidae to be recorded in Vanuatu, Melanesia, southwestern Pacific (Diplopoda: Polydesmida) // Arthropoda Selecta. Vol.17. Nos 3/4. P.145-151.

Golovatch S.I., Geoffroy J.-J, Mauriès J.-P., VandenSpiegel D. 2009. Review of the millipede family Haplodesmidae Cook, 1895, with descriptions of some new or poorly-known species (Diplopoda, Polydesmida) // ZooKeys. Vol.7. P.1-53.

Golovatch S.I., Stoev P. 2009. New or poorly-known millipedes (Diplopoda) from Papua New Guinea, I // Arthropoda Selecta. Vol.18. Nos 3/4. P.125-130.

Golovatch S.I., Stoev P. 2011. Review of the millipede genus Eustrongylosoma Silvestri, 1896 in the island of New Guinea, with descriptions of nine new species (Diplopoda, Polydesmida, Paradoxosomatidae) // International Journal of Myriapodology. Vol.5. P.1-26.

Golovatch S.I., Stoev P. 2013. The millipede family Paradoxosomatidae in the Philippines, with the description of the first, new, Philippine species of Eustrongylosoma Silvestri, 1896, and notes on Anoplodesmus anthracinus Pocock, 1895, recorded in Malaysia and Sri Lanka for the first time (Diplopoda, Polydesmida) // Biodiversity Data Journal. Vol.1.

Hoffman R.L. 1977/1978. Diplopoda from Papuan caves (Zoological Results of the British Speleological Expedition to Papua New Guinea, 1975, 4) // International Journal of Speleology. Vol.9. P.281-307.

Jeekel C.A.W. 1968. On the classification and geographical distribution of the family Paradoxosomatidae (Diplopoda, Polydesmida). Rotterdam, privately published. $162 \mathrm{pp}$.

Jeekel C.A.W. 1973. On the status of the diplopod genus Mimosoma Chamberlin, 1920 (Polydesmida, Paradoxosomatidae) // Bulletin Zoologisch Museum, Universiteit van Amsterdam. Vol.3. No.6. P.31-37.

Jeekel C.A.W. 1980. Records of Diplopoda of the order Polydesmida in the Fiji Islands // Tijdschrift voor Entomologie. Deel 40. S. $122-127$.

Responsible editor K.G. Mikhailov 